\title{
Evaluating Nonpolarizable Nucleic Acid Force Fields: A Systematic Comparison of the Nucleobases Hydration Free Energies and Chloroform-to-Water Partition Coefficients
}

\author{
Maarten G. Wolf* and Gerrit Groenhof
}

Nucleic acid force fields have been shown to reproduce structural properties of DNA and RNA very well, but comparative studies with respect to thermodynamic properties are rare. As a test for thermodynamic properties, we have computed hydration free energies and chloroform-to-water partition coefficients of nucleobases using the AMBER-99, AMBER-gaff, CHARMM-27, GROMOS-45a4/53a6 and OPLS-AA force fields. A mutual force field comparison showed a very large spread in the calculated thermodynamic properties, demonstrating that some of the parameter sets require further optimization. The choice of solvent model used in the simulation does not have a significant effect on the results. Comparing the hydration free energies obtained by the various force fields to the adenine and thymine experimental values showed a very large deviation for the GROMOS and AMBER parameter sets. Validation against experimental partition coefficients showed good agreement for the CHARMM-27 parameter set. In view of mutation studies, differences in partition coefficient between two bases were also compared, and good agreement between experiments and calculations was found for the AMBER-99 parameter set. Overall, the CHARMM-27 parameter set performs best with respect to the thermodynamic properties tested here. (c) 2012 Wiley Periodicals, Inc.

DOI: $10.1002 /$ jcc.23055

\section{Introduction}

Simulations can provide valuable insight into the structure and dynamics of nucleic acids. Large efforts have been made in developing and testing force field parameters to yield structural properties in agreement with experiments. ${ }^{[1-12]}$ However, for a thorough understanding of many biological processes, such as folding, binding, recognition, and so forth, also the partitioning of molecules over different phases is important. However, despite the key role of thermodynamic equilibria for the aforementioned processes, systematic validation studies of nucleic acid force fields are rather scarce. In this study, we have systematically verified the validity of the following force fields with respect to solvation free energies and chloroformto-water partition coefficients of nucleobases: AMBER-99, ${ }^{\text {[13] }}$ AMBER-gaff, ${ }^{[14]}$ CHARMM-27, ${ }^{[15-17]}$ GROMOS-45a4/53a6, ${ }^{[18,19]}$ and OPLS-AA. ${ }^{[20]}$

Hydration free energies ${ }^{[21-28]}$ and chloroform-to-water partition coefficients ${ }^{[29-31]}$ of the nucleobases have been calculated by others, using a variety of computational methods that range from quantum chemistry to classical molecular mechanics (MM) with both explicit and implicit solvent models. However, in these studies, to the best of our knowledge, none of the currently widely used nonpolarizable force fields have been assessed. So far, only the free energy of $\mathrm{G}-\mathrm{C}$ base flipping has been addressed in a previous force field comparison. Although reasonable accuracy was obtained by popular force fields, ${ }^{[32]}$ neither A-T base flipping nor sequence effects were considered.

In this work, we first calculated the hydration free-energy of the nucleobases (Fig. 1). Although only for 9-methyladenine an experimental value and for 1-methylthymine an experimental range $^{[33]}$ are available for comparison, we assessed the hydra- tion free energy for the following reasons; (1) the calculation technique of hydration free energy of small molecules is well established ${ }^{[34-38]}$; (2) the force fields can be directly compared among one another; (3) there is no sequence dependence; (4) small system size ensures fast convergence; (5) the hydrated species is often the reference state in a free energy cycle; (6) the importance of water at the protein-DNA interface. ${ }^{[39,40]}$

Then, to systematically validate the force fields, we calculated nucleobase solvent-to-solvent partition coefficients, for which experimental data is available for all bases. ${ }^{[41,42]}$ From the available experimental partition coefficients we have chosen that of chloroform-to-water for our assessment. This choice was motivated by the fact that the chloroform dielectric constant $\left(4.8^{[43]}\right)$ is similar to the estimated protein dielectric constants (2.5-4 overall for a folded dry protein ${ }^{[44]}$ and $2-20$ at specific sites inside the protein $\left.{ }^{[45]}\right)$. Furthermore, chloroform, in contrast to other solvents that resemble the protein dielectric, such as 2butanol or octanol, does not have slow degrees of freedom such as dihedral angles or hydrogen bonds. Hence, convergence issues with chloroform are expected to be less severe than for instance with 2-butanol or octanol.

Often, one is not interested in removing or adding the base from or to the system, but in the effect of changing one base into another, for example when addressing protein-DNA recognition. To validate the force fields with respect to such

\footnotetext{
M. G. Wolf, G. Groenhof

Computational Biomolecular Chemistry, Max-Planck-Institute for Biophysical Chemistry, Am Faßberg 11, D-37077 Göttingen, Germany

E-mail: mwolf@gwdg.de

Contract/grant sponsor: Alexander von Humbold Stiftung

(c) 2012 Wiley Periodicals, Inc.
} 
<smiles>Cn1cnc2c(N)ncnc21</smiles><smiles>Cc1cn(C(C)C)c(=O)[nH]c1=O</smiles><smiles>CCCCCCn1ccc(=O)[nH]c1=O</smiles><smiles>CN(C)c1ccc(O)cc1</smiles><smiles>Cn1cnc2c(=O)[nH]c(N)nc21</smiles><smiles>Cn1ccc(N)nc1=O</smiles>
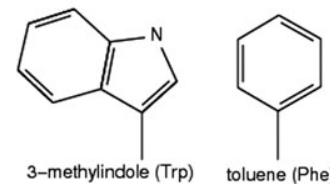

Figure 1. Structure of the nucleobases and aromatic amino acid side chains used in our force field survey. In parentheses the three letter code of the represented nucleic or amino acid.

mutation studies, we also compared the change in the partition coefficients upon base mutation between calculations and experiments. For instance, in protein-DNA recognition, the difference in protein-water partition coefficient determines the order in which, and to what extend, the different bases bind to the protein and, thereby, specificity.

As the nucleobases are conjugated aromatic molecules, we also calculated the same quantities for the aromatic amino acid side chains (Fig. 1). This serves as a consistency check, as for these amino acid analogs both experimental partition coefficients ${ }^{[46]}$ and hydration free energies are available ${ }^{[47]}$ as well as other force field comparison studies. ${ }^{[36,38]}$ In addition, to test the influence of the water model we calculated the hydration free energies and partition coefficients using two popular water models, TIP3P ${ }^{[48]}$ and SPCE. ${ }^{[49]}$ Finally, we discuss the effect of solvent-solvent solubility, which is unavoidable in experiments, but not considered in our calculations.

We note that the molecular polarizability is not explicitly accounted for, due to the fixed point charge description in the MM force fields. ${ }^{[49,50]}$ Because, here, we are interested in testing the performance of the existing nonpolarizable force fields, rather than calculating hydration free energies and partition coefficients as accurately as possible, we accept the limitations of the nonpolarizable simulations and do not apply a posterior correction for the molecular polarizability.

\section{Methods}

All simulations were performed with the Gromacs software package. ${ }^{[51]}$ The simulation boxes consist of one methylated nucleobase or one methylated aromatic amino acid side chain with $\sim 1000$ water molecules or $\sim 250$ chloroform molecules, yielding a solute concentration in water and chloroform of $\sim 55$ and $\sim 50 \mathrm{mM}$, respectively. We used the stochastic dynamics integrator ${ }^{[52]}$ with the reference temperature set to 300 $\mathrm{K}$. Pressure was maintained constant at $1 \mathrm{~atm}$ using the Parrinello Rahman barostat ${ }^{[53]}$ with tau_p set to 1.0 and 5.0 ps and the compressibility to $4.5 \times 10^{-5}$ and $1.086 \times 10^{-4} \mathrm{bar}^{-1}$ for the water and chloroform simulations, respectively. Bond lengths of the solute and of chloroform were constrained using the LINCS algorithm, ${ }^{[54]}$ whereas water molecules were kept completely rigid with the SETTLE algorithm, ${ }^{[55]}$ allowing a time step of $2 \mathrm{fs}$. We used a neighborlist with a cut-off of 1.0, which was updated every five steps. The Lennard Jones interactions were smoothly switched off between 0.8 and $0.9 \mathrm{~nm}$ and a dispersion correction term was added. The electrostatic interactions were treated using $\mathrm{PME}^{[56,57]}$ with a real space cut-off of $1.0 \mathrm{~nm}$. The settings for the long-range interactions are not allways identical to those used during parameterization of the force field, but, as we will later show for the GROMOS force field, the effect is minimal. Furthermore, for the vacuum simulations the Lennard Jones and electrostatic interactions were taken into account between all atom pairs and no periodic boundary conditions were utilized.

For GROMOS-45a4/53a6 calculations were also performed with the simulation set-up used during parametrization of this force field. ${ }^{[19]}$ Accordingly, we used a triple-range approach for the nonbonded interactions, with a short range cut-off of 0.8 $\mathrm{nm}$ and a long range cut-off of $1.4 \mathrm{~nm}$. The pair-list was updated every five steps. The electrostatic interaction outside the long range cut-off were treated using a reaction field ${ }^{[58]}$ with a dielectric constant of 62 and 2.4 for water ${ }^{[19]}$ and chloroform, ${ }^{[59]}$ respectively. Pressure was maintained at $1 \mathrm{~atm}$ using the weak coupling scheme of Berendsen. ${ }^{[60]}$

We calculated the free-energy differences $\Delta G_{\text {sol }}$ and $\Delta G_{\text {vac }}$ that correspond to removing all the nonbonded interactions (Coulomb + Lennard-Jones) of the solute in solvent and in vacuum, respectively (Fig. 2). These free-energy differences were calculated by the Bennett's acceptance ratio ${ }^{[61]}$ based on a discrete $\lambda$-coupling parameter. ${ }^{[62]}$ We used a two-step procedure. First, the solutes electrostatic interactions were turned off using $\lambda$-values $0.00,0.25,0.50,0.75$, and 1.00 , followed by the Lennard-Jones interactions with $\lambda$-values $0.00,0.05,0.10,0.20,0.30$, $0.40,0.50,0.60,0.65,0.70,0.75,0.80,0.85,0.90,0.95$, and 1.00 . Although turning off the Lennard Jones interactions, we used a soft-core potential ${ }^{[63]}$ with an $\alpha$ of 0.5 , a s of $0.3 \mathrm{~nm}$ and a power of 1 . For each $\lambda$ window, we performed a 250 ps equilibration followed by a 1 ns production run.

The calculated free energy differences were converted into solvation free energies (Fig. 2)

$$
\Delta G_{\text {solvation }}=\Delta G_{\mathrm{vac}}-\Delta G_{\mathrm{sol}}
$$

and partition coefficients $\left({ }^{10} \log \left(\frac{c_{\text {water }}}{c_{\text {chloroform }}}\right)\right)$ for the transition from chloroform to water.

$$
{ }^{10} \log \left(\frac{C_{\text {water }}}{c_{\text {chloroform }}}\right)=-\frac{1}{2.303 \cdot R T}\left(\Delta G_{\text {water }}-\Delta G_{\text {chloroform }}\right)
$$

Here, $c$ is the concentration in the specific solvent $(M), R$ the gas constant and $T$ the temperature $(298 \mathrm{~K})$.

We tested five popular force fields in our calculations: AMBER-99, ${ }^{[13,64]}$ AMBER-gaff, ${ }^{[14]}$ CHARMM-27, ${ }^{[15-17,65]}$ GROMOS$45 \mathrm{a} 4$, and $-53 \mathrm{a} 6$ for the nucleobases and amino acids, respectively, ${ }^{[18,19]}$ and OPLS-AA. ${ }^{[20]}$ A methyl group replaces the backbone, for which we assigned charges that are compatible with the force field. For AMBER-99 and CHARMM-27, the hydrogens 


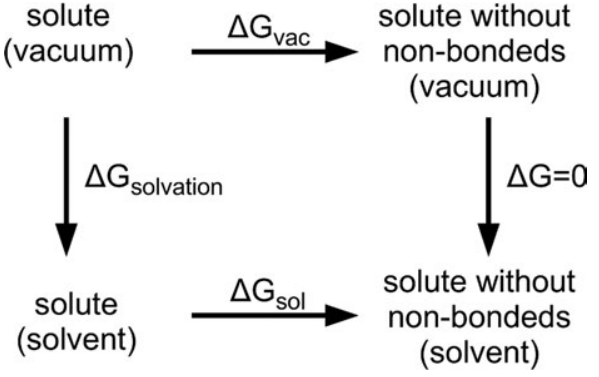

Figure 2. Free energy cycle to calculate solvation free energies $\Delta G_{\text {solvation }}$ The free energy differences $\Delta G_{\text {sol }}$ and $\Delta G_{\text {vac }}$ correspond to removing all solute charge and Lennard Jones interactions in solvent and vacuum, respectively. The solute without nonbonded interactions does not interact with the environment, so that $\Delta G=0$ for the transition from vacuum to solvent.

on the methyl group were given a +0.06 and +0.09 charge, respectively. The carbon was then given a partial charge, such that the whole molecule was neutral. It has been shown that for instance the leucine side chain described with AMBER94, different choices of these charges do not alter the solvation free-energy difference significantly. ${ }^{[38]}$ For the GROMOS-45a4/ $53 a 6$ force field the charge on the methyl group was set to zero and for OPLS-AA the partial charges of a methylated base have been derived elsewhere. ${ }^{[6]}$ For AMBER-gaff the charges were derived to match a quantum mechanical charge distribution (RESP), ${ }^{[14]}$ see Supporting Information. The combination rules of the respective force field were applied to calculate the Lennard-Jones interactions.

Considering the solvent models, we initially calculated the solvation free energy with the solvent model designed for the specific force field. Thus, we used TIP3P ${ }^{[48]}$ for AMBER-99, AMBER-gaff, and CHARMM-27; SPC ${ }^{[67]}$ for GROMOS-45a4/53a6; and TIP4P ${ }^{[48]}$ for OPLS-AA. The internal water structure was constrained using the SETTLE algorithm. ${ }^{[55]}$ The Chloroform parameters were taken from Fox and Kollman ${ }^{[68]}$ for AMBER-99 and AMBER-gaff, from Jørgensen et al. ${ }^{[69]}$ for CHARMM-27 and
OPLS-AA and from Dietz and Heinziger ${ }^{[59,70,71]}$ for GROMOS$45 \mathrm{a} 4 / 53 \mathrm{a} 6$. Finally, we varied the solvent models by calculating the solvation free energies in SPCE and TIP3P for every tested force field and used all chloroform models in combination with the AMBER-99 nucleobases.

\section{Results}

\section{Partition coefficients and solvation free energies}

Validation studies for biomolecular force fields are often performed by calculating solvation free-energies of representative molecules in water. Here, we calculated the solvation free energy of methylated nucleobases and methylated aromatic amino acid side chains in water and chloroform (Tables 1 and 2). After comparing the hydration free energy between the various force fields we see differences of more than $25 \mathrm{~kJ}$ $\mathrm{mol}^{-1}$ in some cases. Especially GROMOS-53a6 deviates significantly from the rest of the tested force fields for Ade, Gua, and Cyt, while the AMBER force fields deviates for Thy and Ura. The results of calculated solvation free energies in chloroform differ much less with a maximum deviation of $10 \mathrm{~kJ}$ $\mathrm{mol}^{-1}$ between the tested force fields.

To ensure that these large differences in solvation free energies between different force fields are actually due to the nucleobase parameters and not the solvent models, we varied the solvent models. The hydration free energies were computed for all force fields with both SPCE and TIP3P water models, and the chloroform solvation free energies were calculated with all three chloroform models in combination with the AMBER-99 force field. The difference in the solvation free energy due to the use of different solvent models is up to $3 \mathrm{~kJ} \mathrm{~mol}^{-1}$ for water and below $1 \mathrm{~kJ} \mathrm{~mol}^{-1}$ for chloroform, which is an order of magnitude smaller than the difference between various force fields, Tables 1 and 2. In addition, for the GROMOS force field we calculated the hydration free energies (and partition coefficients)

Table 1. Calculated solvation free energies $\left(\mathrm{kJ} \mathrm{mol}^{-1}\right)$ of the methylated nucleobases and amino acid side chains in water.

\begin{tabular}{|c|c|c|c|c|c|c|c|c|c|c|}
\hline & Solvent & Ade & Gua & Cyt & Thy & Ura & Trp & Tyr & Phe & $E_{\mathrm{sol}}$ \\
\hline \multirow{2}{*}{ AMBER-99 } & TIP3P & $-58.94(25)$ & $-101.93(37)$ & $-87.33(39)$ & $-63.97(27)$ & $-62.96(23)$ & $-22.86(14)$ & -19.67 (33) & $0.42(24)$ & 5.19 \\
\hline & SPCE & $-59.21(59)$ & $-101.41(36)$ & $-87.09(25)$ & $-63.59(41)$ & $-62.74(44)$ & $-18.73(47)$ & $-16.88(26)$ & $2.78(50)$ & 7.07 \\
\hline \multirow[t]{2}{*}{ AMBER-gaff } & TIP3P & $-64.90(45)$ & $-102.73(48)$ & $-87.98(41)$ & $-56.96(38)$ & $-59.14(39)$ & $-22.25(42)$ & $-17.47(23)$ & $-1.96(20)$ & 5.51 \\
\hline & SPCE & $-64.78(49)$ & $-101.35(31)$ & $-88.40(40)$ & $-56.72(61)$ & $-59.51(28)$ & $-19.30(29)$ & $-14.45(27)$ & 0.39 (34) & 6.81 \\
\hline \multirow[t]{2}{*}{ CHARMM-27 } & TIP3P & $-60.88(52)$ & $-99.78(33)$ & $-81.68(47)$ & $-42.83(25)$ & $-45.57(26)$ & $-16.01(36)$ & $-20.15(48)$ & $-1.42(24)$ & 4.47 \\
\hline & SPCE & $-61.04(49)$ & -99.69 (44) & $-81.97(25)$ & $-40.94(30)$ & $-43.44(42)$ & -14.57 (44) & $-17.44(23)$ & $0.66(38)$ & 5.69 \\
\hline \multirow[t]{4}{*}{ GROMOS-45a4/53a6 } & SPC & $-33.35(36)$ & $-75.78(38)$ & $-66.74(35)$ & $-40.92(37)$ & $-41.93(32)$ & $-26.69(45)$ & $-27.61(22)$ & $-2.41(26)$ & 9.46 \\
\hline & TIP3P & $-34.89(27)$ & -78.15 (44) & $-66.87(43)$ & $-41.56(37)$ & $-42.39(23)$ & $-29.10(30)$ & $-28.51(37)$ & $-3.11(22)$ & 9.02 \\
\hline & SPCE & $-31.24(32)$ & $-76.04(36)$ & $-65.45(42)$ & $-39.58(46)$ & $-40.10(54)$ & $-25.91(27)$ & $-26.27(61)$ & $-0.64(43)$ & 10.29 \\
\hline & $\mathrm{SPC} \mathrm{RF}^{[\mathrm{a}]}$ & $-32.39(30)$ & -75.95 (33) & $-64.90(46)$ & $-39.74(40)$ & $-40.25(32)$ & $-26.82(31)$ & $-26.85(31)$ & $-1.58(24)$ & 12.29 \\
\hline \multirow[t]{3}{*}{ OPLS-AA } & TIP4P & $-54.44(47)$ & $-91.08(36)$ & -78.05 (39) & $-45.85(21)$ & $-44.43(47)$ & $-16.98(40)$ & $-20.36(36)$ & $-1.59(26)$ & 3.90 \\
\hline & TIP3P & $-55.14(25)$ & $-92.64(38)$ & -79.14 (29) & $-46.38(39)$ & $-45.34(26)$ & $-20.10(26)$ & $-22.32(35)$ & $-3.01(30)$ & 2.36 \\
\hline & SPCE & $-53.66(50)$ & $-90.29(50)$ & $-77.59(46)$ & $-44.32(37)$ & $-44.19(38)$ & $-17.56(32)$ & $-21.01(55)$ & $-2.07(30)$ & 3.65 \\
\hline Orozco et al. ${ }^{\left[{ }^{00]}\right.}$ (AM1) & Water & -45.2 & -88.3 & -67.4 & -42.3 & -43.5 & & & & \\
\hline Giesen et al. ${ }^{[29]}$ (SM5.4/A) & Water & -63.6 & -84.1 & -83.3 & -40.2 & -43.9 & & & & \\
\hline $\operatorname{Exp}^{[33,47]}$ & Water & -56.8 & & & $-(38-53)$ & & -24.7 & -25.6 & -3.2 & \\
\hline
\end{tabular}

$E_{\text {sol }}$ is the average absolute error with respect to experiment (if available). Values in parentheses are uncertainties in the last significant digit.

[a] Applying the recommended simulation set-up for the force field. 
Table 2. Calculated solvation free energies $\left(\mathrm{kJ} \mathrm{mol}^{-1}\right)$ of the methylated nucleobases and amino acid side chains in chloroform.

\begin{tabular}{|c|c|c|c|c|c|c|c|c|c|}
\hline & Solvent & Ade & Gua & Cyt & Thy & Ura & Trp & Tyr & Phe \\
\hline \multirow[t]{3}{*}{ AMBER-99 } & Chloroform $^{[68]}$ & $-52.56(22)$ & $-71.76(38)$ & $-58.46(42)$ & $-52.27(47)$ & $-49.42(35)$ & $-36.76(36)$ & $-27.72(24)$ & $-21.44(38$ \\
\hline & Chloroform ${ }^{[69]}$ & $-53.57(30)$ & $-71.79(35)$ & $-58.10(29)$ & $-52.82(29)$ & $-48.77(24)$ & & & \\
\hline & Chloroform ${ }^{[59,70,71]}$ & $-53.21(16)$ & $-71.23(34)$ & $-58.36(63)$ & $-52.32(33)$ & $-49.01(19)$ & & & \\
\hline AMBER-gaff & Chloroform $^{[68]}$ & $-53.66(40)$ & $-69.22(55)$ & $-55.41(45)$ & $-50.55(34)$ & $-47.86(38)$ & $-36.98(23)$ & $-27.58(25)$ & $-21.08(32$ \\
\hline CHARMM-27 & Chloroform $^{[69]}$ & $-58.58(34)$ & $-74.51(51)$ & $-64.40(48)$ & $-48.24(21)$ & $-41.77(32)$ & $-37.46(28)$ & $-28.88(18)$ & $-23.30(35$ \\
\hline GROMOS-45a4/53a6 & Chloroform $[59,70,71]$ & $-45.43(26)$ & $-65.20(43)$ & $-54.74(27)$ & $-47.92(31)$ & -44.00 (19) & $-38.71(36)$ & $-30.32(18)$ & $-22.40(8)$ \\
\hline OPLS-AA & Chloroform ${ }^{[69]}$ & $-56.10(25)$ & $-70.93(36)$ & $-60.69(49)$ & $-57.16(35)$ & $-51.20(29)$ & $-37.11(17)$ & $-29.08(25)$ & $-23.72(27)$ \\
\hline $\operatorname{Exp}^{[a]}$ & Chloroform & -52.3 & & & & & -37.5 & & -16.2 \\
\hline
\end{tabular}

Values in parentheses are uncertainties in the last significant digit.

[a] Obtained by substituting the experimental hydration free energy and chloroform-to-water partition coefficient into eq. (2).

twice. Once with the simulation set-up applied for all force fields and once with the simulation set-up that was used during force field development. ${ }^{[19]}$ The results obtained with these two different simulation set-ups are within $1 \mathrm{~kJ} \mathrm{~mol}^{-1}$. We consider this difference negligible with respect to the deviation from other force fields and the experimental value.

Direct comparison of the nucleobase hydration free energy to experimental values is only possible for Ade. AMBER-99 and OPLS-AA result in the closest agreement to experiment for Ade. For Thy, there is an experimental range available and only the results of the AMBER-99 and AMBER-gaff fall outside this range. When we also consider the aromatic amino acid side chains AMBER-99 and OPLS-AA show the best agreement to experiments. To combine these criteria in one quality measure, we calculated the average absolute error with respect to the experimental value $\left(E_{\text {sol }}\right)$. The average absolute error is obtained by averaging over the absolute value of the difference between the calculated and experimental value (for Thy, an experimental reference value is used that falls within the available experimental range and is closest to the calculated value). A small average absolute error indicates close agreement with experiment over the whole range of tested molecules. The smallest average absolute error is observed for OPLS-AA combined with TIP3P water.

The hydration free energies of the methylated amino acid side chains have been calculated before. ${ }^{[19,36,38]}$ Our calculated values for OPLS-AA show a maximum deviation of $1.6 \mathrm{~kJ} \mathrm{~mol}^{-1}$ to the results of Hess and Van der Vegt ${ }^{[36]}$ or Shirts and Pande. ${ }^{[38]}$ Also, the difference for GROMOS53a6 and AMBER-99 with respect to the results of Hess and Van der Vegt fall within this margin, with the exception of Phe of AMBER-99 (1.7 and 2.4 $\mathrm{kJ} \mathrm{mol}^{-1}$ difference for TIP3P and SPCE, respectively). Small differences like these have been noted before. ${ }^{[36]}$ Note that the difference in hydration free energy of the methylated amino acids as a result of a different water model can be up to $4 \mathrm{~kJ} \mathrm{~mol}^{-1}$.

The limited availability of experimental nucleobase hydration free-energies demands another thermodynamic quantity to validate the force fields against experiments. Therefore, we calculated chloroform-to-water partition coefficients. The calculated partition coefficients are listed in Table 3 together with the results from previous calculations by other groups and the experimental values. We also calculated the chloroform-to-water partition coefficients for the three aromatic amino acid side chains.
We observed a significant spread in the partition coefficients calculated with the different force fields, Table 3. Compared to experimental data, the OPLS-AA force field performs excellent for the Cyt and Gua bases, but poorly for Ade, Thy, and Ura. GROMOS-53a6 shows very good agreement for Trp, which has also been demonstrated for a modified version of GROMOS-87, ${ }^{\text {[72] }}$ but for all other residues the discrepancy between the GROMOS$45 \mathrm{a} 4 / 53 \mathrm{a} 6$ and experimental results is larger than with the other force fields. Furthermore, we notice that in several cases the sign of the calculated partition coefficient is opposite to that of the experimental value. This means that in simulations the solute prefers a distinct solvent than in experiment, and, thus, the partition behavior in experiments and simulations is different.

We calculated average absolute errors between the calculated and experimental partition coefficients. The following average absolute errors were calculated: $\left(E_{\mathrm{NA}}\right)$ considering only nucleobases; $\left(E_{\text {tot }}\right)$ considering both nucleobases and aromatic amino acid side chains; and $\left(E_{\text {mut }}\right)$ the average absolute error upon base mutation for all possible nucleobase mutations. For $E_{\mathrm{NA}}$ we found that CHARMM-27 performs best with an average absolute error of 0.65 in the chloroform-to-water partition coefficient or $3.73 \mathrm{~kJ} \mathrm{~mol}^{-1}$ in $\Delta \Delta G$. Including the results of the aromatic amino acid side chains $\left(E_{\text {tot }}\right)$ did not change the force field ranking significantly. In contrast, for AMBER-99 and GROMOS-45a4/53a6, we observed closest agreement with experiment for the difference in partition coefficient between two bases (smallest $E_{\text {mut }}$ ). For these force fields, every nucleobase shows the same sign for the deviation from the experimental value and the difference in partition coefficient between two bases benefits from cancellation of error.

To graphically illustrate force field performance, the computed partition coefficients are plotted against the experimental values in Figure 3. In this plot, points above the $y=x$ line mean that the force field predicts a larger fraction in the water phase than found experimentally, whereas points below this line indicate that the chloroform phase is more favoured over the water phase than found in experiments. Regarding the methylated nucleobases, Figure 3 shows that the AMBER force fields have an increased affinity for the water phase over the chloroform phase, whereas for GROMOS-45a4/53a6 the opposite is true. Values for CHARMM-27 and OPLS-AA are found on both sides of the diagonal.

Qualitative studies on nucleic acid recognition, drug binding, and so forth, may require a good representation of the relative 
Table 3. Calculated chloroform-to-water partition coefficients $\left[\log \left(c_{\text {water }} / c_{\text {chloroform }}\right)\right]$ for different water models.

\begin{tabular}{|c|c|c|c|c|c|c|c|c|c|c|c|c|}
\hline & Solvent-solvent & Ade & Gua & Cyt & Thy & Ura & Trp & Tyr & Phe & $E_{N A}$ & $E_{\text {tot }}$ & $E_{m u t}$ \\
\hline \multirow[t]{2}{*}{ AMBER-99 } & TIP3P-Chloroform & $1.12(1)$ & $5.28(4)$ & $5.06(4)$ & $2.05(4)$ & $2.37(2)$ & $-2.43(2)$ & $-1.41(2)$ & $-3.83(3)$ & 1.38 & 1.24 & 0.81 \\
\hline & SPCE-Chloroform & $1.16(6)$ & $5.19(3)$ & $5.01(3)$ & $1.98(5)$ & $2.33(4)$ & $-3.16(5)$ & $-1.90(2)$ & $-4.24(5)$ & 1.35 & 1.37 & 0.76 \\
\hline \multirow[t]{2}{*}{ AMBER-gaff } & TIP3P-Chloroform & $1.97(5)$ & $5.87(7)$ & $5.70(5)$ & $1.12(3)$ & $1.98(4)$ & $-2.58(3)$ & $-1.77(1)$ & $-3.35(2)$ & 1.54 & 1.30 & 1.13 \\
\hline & SPCE-Chloroform & $1.95(5)$ & $5.63(6)$ & $5.78(5)$ & $1.08(7)$ & $2.04(3)$ & $-3.10(2)$ & $-2.30(2)$ & $-3.76(3)$ & 1.50 & 1.41 & 1.12 \\
\hline \multirow[t]{2}{*}{ CHARMM-27 } & TIP3P-Chloroform & $0.40(5)$ & $4.43(5)$ & $3.03(6)$ & $-0.95(1)$ & $0.67(2)$ & $-3.76(3)$ & $-1.53(4)$ & $-3.83(2)$ & 0.65 & 0.90 & 1.04 \\
\hline & SPCE-Chloroform & $0.43(5)$ & $4.41(6)$ & $3.08(4)$ & $-1.28(2)$ & $0.29(4)$ & $-4.01(4)$ & $-2.00(1)$ & $-4.20(3)$ & 0.79 & 1.09 & 1.25 \\
\hline \multirow[t]{3}{*}{ GROMOS-45a4/53a6 } & SPC-Chloroform & $-2.12(3)$ & $1.85(4)$ & $2.10(3)$ & $-1.23(3)$ & $-0.36(2)$ & $-2.11(4)$ & $-0.47(1)$ & $-3.50(1)$ & 1.74 & 1.44 & 0.82 \\
\hline & TIP3P-Chloroform & $-1.85(2)$ & $2.27(5)$ & $2.12(4)$ & $-1.11(3)$ & $-0.28(1)$ & $-1.68(3)$ & $-0.32(3)$ & $-3.38(1)$ & 1.56 & 1.35 & 0.76 \\
\hline & SPCE-Chloroform & $-2.49(2)$ & $1.90(4)$ & $1.88(3)$ & $-1.46(4)$ & $-0.68(5)$ & $-2.24(3)$ & $-0.71(7)$ & $-3.81(3)$ & 1.96 & 1.62 & 0.92 \\
\hline \multirow[t]{3}{*}{ OPLS-AA } & TIP4P-Chloroform & $-0.29(4)$ & $3.53(3)$ & $3.04(5)$ & $-1.98(2)$ & $-1.19(4)$ & $-3.53(3)$ & $-1.53(3)$ & $-3.88(2)$ & 1.19 & 1.26 & 1.47 \\
\hline & TIP3P-Chloroform & $-0.17(2)$ & $3.80(3)$ & $3.23(4)$ & $-1.89(3)$ & $-1.03(2)$ & $-2.98(1)$ & $-1.18(2)$ & $-3.63(2)$ & 1.21 & 1.16 & 1.54 \\
\hline & SPCE-Chloroform & $-0.43(5)$ & $3.39(5)$ & $2.96(6)$ & $-2.25(3)$ & $-1.23(3)$ & $-3.42(2)$ & $-1.41(6)$ & $-3.79(2)$ & 1.30 & 1.32 & 1.53 \\
\hline Orozco et al. ${ }^{[30]}\left(6-31 G^{*}\right)$ & & -1.3 & 2.0 & 1.9 & 0.1 & 0.7 & & & & 1.1 & & 0.9 \\
\hline Orozco et al. ${ }^{[30]}$ (AM1) & & 0.3 & 4.8 & 3.4 & 0.4 & 1.0 & & & & 0.5 & & 0.8 \\
\hline Giesen et al. ${ }^{[29]}$ (SM5.4/A) & & 1.4 & 3.1 & 3.2 & 0.3 & 1.2 & & & & 0.3 & & 0.5 \\
\hline $\begin{array}{l}\text { Eksterowicz et al. }{ }^{[31]} \\
\text { (AMBER-94) }\end{array}$ & & -1.8 & 1.6 & 2.0 & -0.5 & -0.1 & & & & 1.6 & & 0.8 \\
\hline $\operatorname{Exp}^{[41,46]}$ & & 0.78 & 3.52 & 3.00 & 0.45 & 1.21 & -2.24 & & -2.28 & & & \\
\hline
\end{tabular}

$E_{N A}, E_{\text {tot }}, E_{\text {mut }}$ are the average absolute error for nucleic acids only, all listed values and all possible combinations of partition coefficients difference

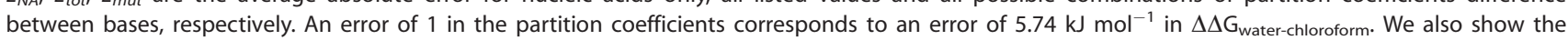
values from other groups and experiments (errors were not given). Values in parentheses are uncertainties in the last significant digit.

hydrophobicity of the nucleobases. The relative hydrophobicity of molecules is conveniently expressed on a hydrophobicity scale, with more hydrophobic molecules higher up the scale. For nucleobases, it has been shown that the chloroform-to-water partition coefficient ${ }^{[41]}$ shows the same trend as the traditional hydrophobicity scale based on the cyclohexane-to-water partitioning. ${ }^{[42]}$ The hydrophobicity scale can be extracted from Table 3 , where a higher value of the partition coefficient means that the molecule is more hydrophobic. The hydrophobicity scale is illustrated in Figure 4. The scale based on the calculated partition coefficients differs from the experimental scale for all force fields except CHARMM-27. With respect to relative hydrophobicity,

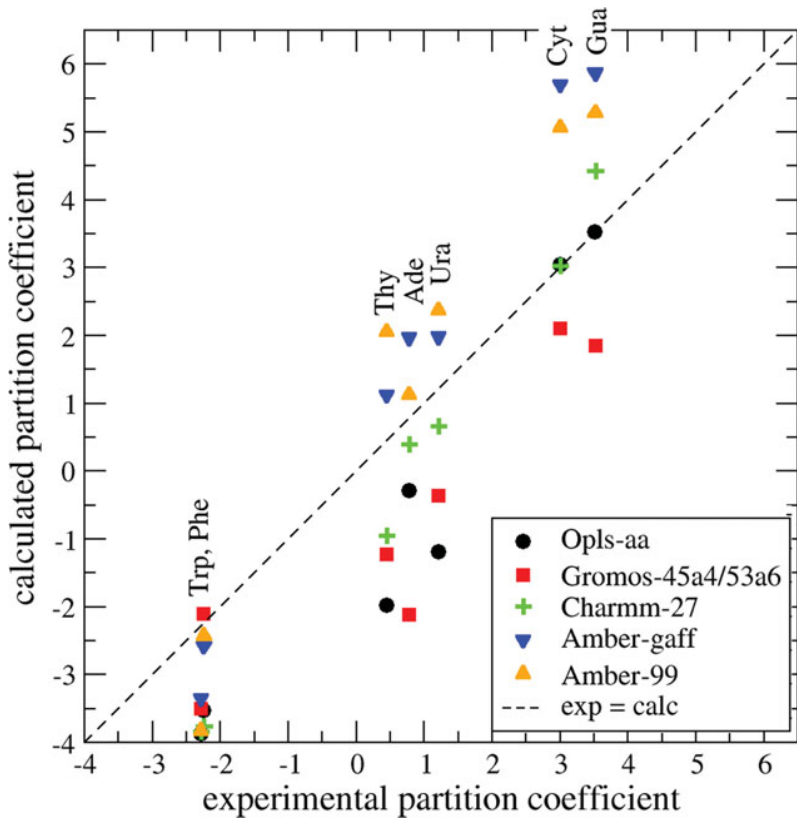

Figure 3. Correlation between the calculated chloroform-to-water partition coefficients $\left(\log \left(c_{\text {water }} / c_{\text {chloroform }}\right)\right)$ and the experimental value.
CHARMM-27 thus yields the best agreement with experiments. The mismatch between force field and experimental scale is mostly found in the position of Ade with respect to Thy and/or Ura, but in case of GROMOS-45a4/53a6 also the position of Cyt and Gua is exchanged. Calculations by other groups show similar errors in the hydrophobicity scale. ${ }^{[29-31]}$ The hydrophobicity scale is very sensitive, a difference of $0.33-0.52$ in the partition coefficient difference between two bases would be sufficient to alter the order of Ade and Thy/Ura; or Cyt and Gua.

\section{Water model}

The majority of biomolecular simulations address aqueous systems. Hence, the interaction between the biomolecules as described by a specific force field and the water model is extremely important. Therefore, we calculated the hydration free energies and partition coefficients of the nucleobases with two widely used water models, SPCE ${ }^{[49]}$ and TIP3P. ${ }^{[48]}$

In general, the hydration free-energies calculated with SPCE are a little higher than those calculated with TIP3P (on average $1.56 \mathrm{~kJ} \mathrm{~mol}^{-1}$ ). The value obtained with the SPC and TIP4P water model with GROMOS-45a4/53a6 and OPLS-AA, respectively, fall mostly between the TIP3P and SPCE value. A similar trend is observed when the hydration free energies of amino acids analogues are calculated using various water models. ${ }^{[36,37]}$ The difference in hydration free energy due to the use of a different water model is larger in the case of the aromatic amino acid analogues than the nucleobases. The overall agreement in hydration free energy between calculations and experiment, as measured by $E_{\text {sol, }}$ is highest with the TIP3P water model.

The partition coefficients calculated with the water model native to the force fields, as well as the TIP3P and SPCE water model are shown in Table 3. The average absolute errors $E_{\mathrm{NA}}$ and $E_{\text {tot }}$ show that for the AMBER force fields the SPCE and TIP3P water model result in similar errors. For CHARMM-27, GROMOS45a4/53a6, and OPLS-AA, the best agreement with experiments 


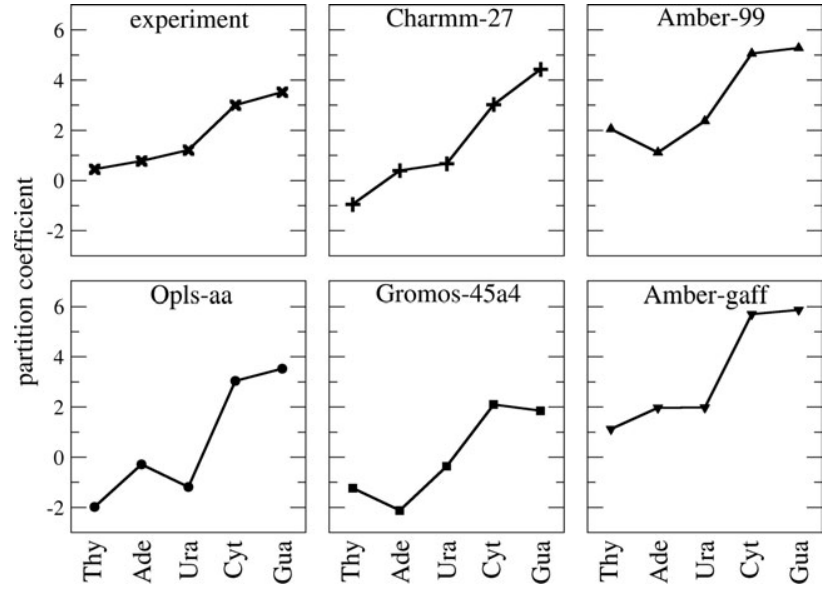

Figure 4. Nucleobase hydrophobicity scale. On the $x$-axis, the nucleobases are ordered according to their experimental hydrophobicity. A (partly) declining line means that the respective hydrophobicity scale deviates from the experimental. For each force field only the results for the native solvent model are shown, the other tested solvent models yielded very similar graphs.

is obtained with the TIP3P water model. However, it is unclear what the effect of the chloroform model is in this comparison.

The water model also affects the predicted hydrophobicity scale and changes the order in some cases. For example, the position of Gua and Cyt on the hydrophobicity scale calculated with the AMBER-gaff force field is different with the TIP3P or SPCE water model (correct and incorrect position, respectively). When considering the influence of different water models on the difference in partition coefficients between two bases, as measured by $E_{\text {mut }}$, we do not find a general trend.

\section{Solvent-solvent solubility}

An important difference between the experimental and our simulation set up is the solvent-solvent contact. The experimental measurements are performed with a two-phase system, where the solvents can dissolve into each other. ${ }^{[41]}$ In contrast, our calculations are performed on two one-phase systems without solvent-solvent contact. To address the influence of solvent-solvent solubility on the calculated thermodynamic properties, we have performed simulation with one molecule of the other solvent added to each one-phase system. This approach is only a rough approximation to the real solvent-solvent solubility, omiting the possibility that clusters of one solvent can exist in the other solvent. Furthermore, the free energies and partition coefficients calculated this way suffer considerably from convergence problems. Therefore, with this approach the effect of solvent-solvent solubility can only be interpreted in general terms.

The computed partition coefficients and solvation free energies for which we have taken the solvent-solvent solubility into account are provided as supplementary information, because the values are not quantitative. The general trend observed from our calculations is a decrease of the solvation free energy in chloroform, leading to a decrease of the partition coefficient. As the partition coefficients predicted with the AMBER force fields are generally too high the agreement with experiments is improved. Conversely, the partition coefficients obtained with the other force fields are mostly already smaller than the experimental value, and agreement with experiments for these force fields gets worse.

\section{Discussion}

We have assessed various popular nucleic acid force fields for their ability to yield correct hydration free energies and chloroform-to-water partition coefficients. In this investigation, we have tested the following force fields: AMBER-99, ${ }^{[13]}$ AMBER-gaff, ${ }^{[14]}$ CHARMM-27, ${ }^{[15-17]}$ GROMOS-45a4/53a6, ${ }^{[18,19]}$ and OPLS-AA. ${ }^{[20]}$ We addressed the nucleobase parameters by comparing simulations mutually and to experiments on three criteria: (1) hydration free energy, (2) chloroform-to-water partition coefficients, (3) difference in partition coefficients between two bases (mutation).

Comparing calculated hydration free energies to experimental values is a common and convenient approach to test force field accuracy. Unfortunately, for nucleobases, the experimental data are limited to 9-methyladenine and a range for 1-methylthymine. The large negative hydration free energies of 1-methylcytosine and 9-methylguanine that we obtained agree with the low volatility that prohibits experimental determination of hydration free energies. ${ }^{[41]}$ Within this limited framework, OPLS-AA showed good agreement to experiments. However, a very large deviation with experiments was found for GROMOS-45a4 9-methyladenine and AMBER-99 1-methylthymine and 1-methyluracil parameters. The large spread in calculated values obtained from various force fields is reason for concern. The tests with two different simulation set-ups for GROMOS-53a6 as well as different water models for all force fields and different chloroform models with AMBER-99 indicate that mainly the different nucleobase parameters are responsible for this large spread.

Experimental thermodynamic data for all nucleobases are available when we consider chloroform-to-water partition coefficients. ${ }^{[41,42]}$. An additional advantage of this quantity is that chloroform has a similar dielectric constant as a protein environment. A straightforward comparison of the calculated and experimental partition coefficients shows the best agreement for CHARMM-27. When solvent-solvent solubility is taken into account by adding one molecule of the other solvent to the 1-phase system, the average absolute error for CHARMM-27 increases slightly and AMBER-99 now performs equally well.

The average disagreement between experiments and calculations (average absolute error) is in the range of 1 or $4.18 \mathrm{~kJ}$ $\mathrm{mol}^{-1}$, which suggests that the distribution of a nucleic acid base between the water phase and a protein environment will be sampled with reasonable accuracy by these two force fields. Obviously, our assessment does not include the nucleic acid backbone. Also, extrapolating our results to specific nucleic-acid protein configurations is difficult, because the sequence specific interactions, amino acid to nucleobase hydrogen bonding and excluded volume effects are not taken into account. Nevertheless, it is encouraging to find that the nonspecific interactions governing partitioning between water and a solvent with low dielectric are reproduced reasonably well with CHARMM-27 and AMBER-99.

Mutation studies are a common experimental approach to assess nucleic acid-protein interactions. To address how well 
nucleic acid mutations can be represented in simulations, we analyzed how well the change in partition coefficients upon base mutation (the difference in partition coefficient between two bases) is reproduced with the different parameter sets. Qualitatively, only CHARMM-27 performs satisfactorily, as it is the only force field that predicts the hydrophobicity scale (or the sign of the partition coefficient difference between bases) correctly. Quantitatively, AMBER-99 shows the best agreement with experiments. This indicates that, although CHARMM-27 might predict the sign of a mutation correctly the average deviation from experiments is large. In contrast, AMBER-99 results in a reasonably small deviation from experiments in the mutation free energy (average absolute error $E_{\text {mut }}$ ), but occasionally result in the wrong sign. It should be noted that specific interactions, such as specific hydrogen bonds, might compensate sufficiently for these force field inaccuracies to yield qualitatively correct results in studies of for instance proteinDNA binding, recognition, and so forth, but these aspects were outside the scope of our study.

Comparing our results to partition coefficients previously obtained by others using different computational methods, shows that a better match with experiments is obtained when using semiempirical quantum chemistry approaches with implicit solvent models. ${ }^{[30,29]}$ However, questions concerning DNA binding, recognition, and so forth, are commonly addressed by classical MD simulations using MM force fields, because semiempirical methods are computationally prohibitively expensive. In addition, the agreement with experiment of the hydration free energy for these semiempirical methods is not as good as in case of the tested force fields (Table 1). Also, the mutual agreement between the two semiempirical methods is poor (Tables 1 and 3). Notably, a full quantum description including a continuum solvent ${ }^{[30]}$ shows comparable agreement with experiments as the force fields tested here.

The choice of water and chloroform model can potentially influence the outcome of the free-energy calculations and, therefore, also the partition coefficients. This has already been shown with respect to amino acid analogues regarding the water models. ${ }^{[36,37]}$ Here, we tested the use of the SPCE and TIP3P water models for all force fields in our survey. These two popular models have low computational costs and are thus attractive when very long simulation times are required, for example, for free-energy calculations. On average, the results with the TIP3P model show the best agreement with experiments for all tested force fields for the hydration free energy. However, to put this into perspective, the average difference between values calculated with TIP3P and SPCE waters is within $1.56 \mathrm{~kJ} \mathrm{~mol}^{-1}$, which is much smaller than the average deviation from the experimental value $\left(2.36-12.29 \mathrm{~kJ} \mathrm{~mol}^{-1}\right.$ ) or the difference between different force fields (up to $30 \mathrm{~kJ}$ $\mathrm{mol}^{-1}$ ). Furthermore, experimental hydration free energies for the Cyt, Gua, and Ura bases are not available, making the test of the water models based on hydration free energies incomplete. TIP3P also yields the best agreement between experiments and calculations for the chloroform-to-water partition coefficient, but, as for the hydration free energy, the differences between water models are much smaller than the differen- ces between the other force fields or the experiments. In addition, there may be cancellation of error as a result of the chloroform model. In conclusion, the small differences in both the hydration free energies and the partition coefficients as a result of distinct water models in combination with the uncertainties discussed in this paragraph, we cannot conclusively recommend one of the tested water models.

A potentially critical issue when comparing experimental and computed partition coefficients is solvent-solvent solubility. During experiments there is always a (tiny) fraction of one solvent dissolved in the other. As a rough approximation, we have tried to address this aspect qualitatively, by calculating partition coefficients with one water added to the chloroform phase and one chloroform added to the water phase. We observe a small decrease in almost all calculated partition coefficients. The shift of the partition coefficient due to solvent-solvent solubility is mainly due to one water added to the chloroform phase (see Supporting Information Table Si). A possible explanation may be that water, with its higher dielectric constant, can shield dissolved polar molecules much better than chloroform. Consequently interaction with another dissolved (polar) molecule will be weaker in water than in chloroform. An additional polar molecule in the chloroform phase will thus have a higher impact on the free energy of solvation than an additional nonpolar molecule in the water phase. This is exactly what we observe for the computed solvation freeenergies with one molecule of the other solvent (see Supporting Information Table Si). Taking solvent-solvent solubility into account improves the agreement with experiments for the AMBER force fields, but decreases it for the other force fields.

Note that due to the sampling bottleneck as well as the inability to dynamically alter the number of different solvent molecules, our calculations cannot be used for a quantitative assessment of solvent-solvent solubility. In an attempt to obtain a quantitative measure, we have simulated an adenine base in a water-chloroform two phase system for 5 ms using AMBER-99. However, despite the considerable computational effort, the density profile, and hence, also the free energy profile, was not converged yet. At the current state of the art, this approach is thus prohibitively expensive for a force field comparison.

\section{Conclusion}

We have calculated nucleobase hydration free energies and chloroform-to-water partition coefficients for five popular MM force fields and found the best performance with CHARMM-27. The nucleobase hydration free energies showed a large spread among the various force fields tested and especially the GROMOS and AMBER parameter sets did not agree well to the few available experimental values. With respect to the chloroform-to-water partition coefficient, the best agreement to experiments is obtained with CHARMM-27. If we consider base mutations, AMBER-99 yields closest agreement between experiments and calculations.

Based on the tested thermodynamic properties here and the structural studies performed by other groups ${ }^{[4-6,8,9]}$ we conclude that CHARMM-27 is currently reproducing experimental data the best. The AMBER-99 force field is potentially a 
good alternative, provided that the disagreement between calculated and measured hydration free energies of thymine and uracil can be resolved.

\section{Acknowledgments}

The authors thank David van der Spoel and Berk Hess for useful discussion and Bert de Groot for carefully reading the manuscript.

Keywords: CHARMM - AMBER - GROMOS - OPLS-AA - GROMACS - molecular dynamics - free energy - nucleobases · solvation $\cdot$ thermodynamics $\cdot$ DNA $\cdot$ RNA $\cdot$ partition coefficient

How to cite this article: M. G. Wolf, G. Groenhof, J. Comput. Chem. 2012, 33, 2225-2232. DOI: 10.1002/jcc.23055

Additional Supporting Information may be found in the online version of this article.

[1] S. Reddy, F. Leclerc, M. Karplus, Biophys. J. 2003, 84, 1421.

[2] T. Cheatham, M. Young, Biopolymers 2001, 56, 232.

[3] C. Oostenbrink, T. Soares, N. van der Vegt, van Gunsteren, W., Eur. Biophys. J. 2005, 34, 273

[4] A. Pérez, F. Lankas, F. Luque, M. Orozco, Nucleic Acids Res. 2008, 36, 2379.

[5] C. Ricci, A. de Andrade, M. Mottin, P. Netz, J. Phys. Chem. B 2010, 114 9882.

[6] N. Foloppe, L. Nilsson, J. Phys. Chem. B 2005, 109, 9119.

[7] E. Fadrná, N. Spacková, J. Sarzyñska, J. Koca, M. Orozco, T. Cheatham, T. Kulinski, J. Sponer, J. Chem. Theory Comput. 2009, 5, 2514.

[8] P. Banáš, D. Hollas, M. Zgarbová, P. Jurečka, M. Orozco, T. Cheatham III, J. Sponer, M. Otyepka, J. Chem. Theory Comp. 2010, 6, 3836.

[9] Beššeová, I.; M. Otyepka, K. Réblová, J. Šponer, Phys. Chem. Chem. Phys. 2009, 11, 10701.

[10] R. Lavery, K. Zakrzewska, D. Beveridge, T. C. Bishop, D. Case, T. Cheatham, S. Dixit, B. Jayaram, F. Lankas, C. Laughton, J. H. Maddocks, A Michon, R. Osman, M. Orozco, A. Perez, T. Singh, N. Spackova, J. Sponer, Nucleic Acids Res. 2010, 38, 299.

[11] T. Gaillard, D. Case, J. Chem. Theor. Comput. 2011, 7, 3181.

[12] B. Jayaram, D. Sprous, M. Young, D. Beveridge, J. Am. Chem. Soc. 1998 120, 10629.

[13] J. Wang, P. Cieplak, P. A. Kollman, J. Comput. Chem. 2000, 21, 1049.

[14] J. Wang, R. Wolf, J. Caldwell, P. Kollman, D. Case, J. Comput. Chem., 2004, 25, 1157

[15] A. D. MacKerell, N. Banavali, N. Foloppe, Biopolymers 2000, 56, 257.

[16] A. D. MacKerell, N. K. Banavali, J. Comput. Chem. 2000, 21, 105.

[17] N. Foloppe, A. D. MacKerell, Jr., J. Comput. Chem. 2000, 21, 86.

[18] T. Soares, P. Hünenberger, M. Kastenholz, V. Kräutler, T. Lenz, R. Lins, C. Oostenbrink, W. Van Gunsteren, J. Comput. Chem. 2005, 26, 725.

[19] C. Oostenbrink, A. Villa, A. Mark, W. van Gunsteren, J. Comput. Chem. 2004, 25, 1656

[20] G. Kaminski, R. Friesner, J. Tirado-Rives, W. Jorgensen, J. Phys. Chem. B 2001, 105, 6474.

[21] J. Miller, P. Kollman, J. Phys. Chem. 1996, 100, 8587.

[22] J. Gao, Biophys. Chem. 1994, 51, 253.

[23] A. Elcock, W. Richards, J. Am. Chem. Soc. 1993, 115, 7930.

[24] P. Young, I. Hillier, Chem. Phys. Lett. 1993, 215, 405.

[25] P. Bash, U. Singh, R. Langridge, P. Kollman, Science 1987, 236, 564.

[26] C. Cramer, D. Truhlar, Chem. Phys. Lett. 1992, 198, 74.

[27] V. Mohan, M. Davis, J. McCammon, B. Pettitt, J. Phys. Chem. 1992, 96, 6428.

[28] M. Orozco, F. Luque, Biopolymers 1993, 33, 1851
[29] D. Giesen, C. Chambers, C. Cramer, D. Truhlar, J. Phys. Chem. B 1997 101, 5084.

[30] M. Orozco, C. Colominas, F. Luque, Chem. Phys. 1996, 209, 19.

[31] J. Eksterowicz, J. Miller, P. Kollman, J. Phys. Chem. B 1997, 101, 10971.

[32] U. Priyakumar, A. MacKerell, J. Comput. Theory Chem. 2006, 2, 187.

[33] D. Ferguson, D. Pearlman, W. Swope, P. A. Kollman, J. Comput. Chem. 1992, 13, 362.

[34] D. Mobley, C. Bayly, M. Cooper, M. Shirts, K. Dill, J. Chem. Theor. Comput. 2009, 5, 350 .

[35] D. Mobley, C. Bayly, M. Cooper, K. Dill, J. Phys. Chem. B 2009, 113, 4533.

[36] B. Hess, N. Van der Vegt, J. Phys. Chem. B 2006, 110, 17616.

[37] M. Shirts, V. Pande, J. Chem. Phys. 2005, 122, 134508.

[38] M. Shirts, J. Pitera, W. Swope, V. Pande, J. Chem. Phys. 2003, 119, 5740.

[39] B. Jayaram, T. Jain, Ann. Rev. Biophys. Biomol. Struct. 2004, 33, 343.

[40] C. Reddy, A. Das, B. Jayaram, J. Mol. Biol. 2001, 314, 619.

[41] P. Cullis, R. Wolfenden, Biochemistry 1981, 20, 3024.

[42] P. Shih, L. Pedersen, P. Gibbs, R. Wolfenden, J. Mol. Biol. 1998, 280, 421.

[43] Permittivity (Dielectric Constant) of Liquids, in CRC Handbook of Chemistry and Physics, 92nd Edition (Internet Version 2012), W. M. Haynes, ed., CRC Press/Taylor and Francis, Boca Raton, FL.

[44] M. Gilson, B. Honig, Biopolymers 1986, 25, 2097.

[45] G. King, F. Lee, A. Warshel, J. Chem. Phys. 1991, 95, 4366.

[46] A. Radzicka, R. Wolfenden, Biochemistry 1988, 27, 1664.

[47] R. Wolfenden, L. Andersson, P. Cullis, C. Southgate, Biochemistry 1981, 20, 849 .

[48] W. Jorgensen, J. Chandrasekhar, J. Madura, R. Impey, M. Klein, J. Chem. Phys. 1983, 79, 926

[49] H. Berendsen, J. Grigera, T. Straatsma, J. Phys. Chem. 1987, 91, 6269.

[50] C. Böttcher, Theory of Electric Polarization, Vol. 1, 2nd ed.; Amsterdam: Elsevier scientific publishing company, 1973.

[51] B. Hess, C. Kutzner, D. van der Spoel, E. Lindahl, J. Chem. Theor. Comput. 2008, 4, 435.

[52] W. Van Gunsteren, H. Berendsen, Mol. Simul. 1988, 1, 173.

[53] M. Parrinello, A. Rahman, J. Appl. Phys. 1981, 52, 7182.

[54] B. Hess, H. Bekker, H. J. C. Berendsen, J. G. E. M. Fraaije, J. Comput. Chem. 1997, 18, 1463.

[55] S. Miyamoto, P. A. Kollman, J. Comput. Chem. 1992, 18, 1463.

[56] T. Darden, D. York, L. Pedersen, J. Chem. Phys. 1993, 98, 10089.

[57] U. Essmann, L. Perera, M. Berkowitz, T. Darden, H. Lee, L. Pedersen, J. Chem. Phys. 1995, 103, 8577.

[58] I. Tironi, R. Sperb, P. Smith, W. van Gunsteren, J. Chem. Phys. 1995 102, 5451.

[59] I. Tironi, W. Van Gunsteren, Mol. Phys. 1994, 83, 381.

[60] H. Berendsen, J. Postma, W. van Gunsteren, A. D. Nola, J. Haak, J. Chem. Phys. 1984, 81, 3684.

[61] C. Bennett, J. Comput. Phys. 1976, 22, 245.

[62] J. Kirkwood, J. Chem. Phys. 1935, 92, 7570.

[63] T. Beutler, A. Mark, R. Van Schaik, P. Greber, W. Van Gunsteren, Chem. Phys. Lett. 1994, 222, 529.

[64] E. J. Sorin, V. S. Pande, Biophys. J. 2005, 88, 2472.

[65] P. Bjelkmar, P. Larsson, M. Cuendet, B. Hess, E. Lindahl, J. Chem. Theory Comput. 2010, 6, 459.

[66] J. Pranata, S. Wierschke, W. Jorgensen, J. Am. Chem. Soc. 1991, 113, 2810.

[67] H. J. C. Berendsen, J. P. M. Postma, W. F. van Gunsteren, J. Hermans, In Pullman Editors Intermolecular Forces; D. Reidel Publishing Company: Dordrecht, 1981; pp. 331-342.

[68] T. Fox, P. Kollman, J. Phys. Chem. B 1998, 102, 8070

[69] W. Jorgensen, J. Briggs, M. Contreras, J. Phys. Chem. 1990, 94, 1683.

[70] W. Dietz, K. Heinziger, Ber. Bunsen-Ges. Phys. Chem. 1984, 88, 543.

[71] Dietz, W. and Heinziger, K., Ber. Bunsen-Ges. Phys. Chem., 1985, 89, 968.

[72] X. Daura, P. Hünenberger, A. Mark, E. Querol, F. Avilés, W. van Gunsteren, J. Am. Chem. Soc. 1996, 118, 6285294.

Received: 27 February 2012

Revised: 29 May 2012

Accepted: 4 June 2012

Published online on 10 July 2012 